\title{
Digital Signal Reconstruction in the ATLAS Hadronic Tile Calorimeter
}

\author{
E. Fullana, J. Castelo, V. Castillo, C. Cuenca, A. Ferrer, E. Higon, C. Iglesias, A. Munar, J. Poveda, \\ A. Ruiz-Martinez, B. Salvachua, C. Solans, R. Teuscher, and J. Valls
}

\begin{abstract}
We present an Optimal Filtering (OF) algorithm to reconstruct the energy, time and pedestal of a photomultiplier signal from its digital samples. The $O F$ algorithm was first developed for liquid ionization calorimeters, its implementation in scintillator calorimeters, specifically in the ATLAS hadronic Tile calorimeter (TileCal), is the aim of this study. The objective is to implement the algorithm on the DSPs of the Read Out Driver cards in order to reconstruct online the energy of the calorimeter and provide it to the second level trigger. The algorithm is tested and compared with a plain filtering algorithm using both calibration and real data from the TileCal detector. The results are promising specially in the regions where the electronic noise contributes significantly to the resolution.
\end{abstract}

\section{INTRODUCTION}

$\mathbf{T}$ HE Hadronic Tile Calorimeter (TileCal) is the central hadronic calorimeter of the ATLAS detector [1]. It is a sampling calorimeter made of iron as passive material and plastic scintillators as active material. The light produced in the scintillators is send to photomultipliers by wave-length shifting fibers. The photomultipliers (PMs) produce an electrical signal which is shaped and digitized by the front-end electronics. The digital samples of the signal are transmitted to the Read Out Driver (ROD) boards through optical fibers. The ROD system reconstructs the energy of all the channels of the TileCal detector during the first level trigger latency of the ATLAS detector, which is $10 \mu \mathrm{s}$. Once the energy is reconstructed, it is sent to the second level trigger. There are $\sim 10000$ channels in the TileCal detector and seven samples of 10 bits are taken per channel in each event. The ROD receives the events once they are accepted by the first level trigger, i.e., every $10 \mu \mathrm{s}$. Therefore the ROD system must process up to $80000 \mathrm{Mb} / \mathrm{s}$. In order to do that there are 32 ROD motherboards divided in 4 partitions. Each motherboard is equipped with two processing units each with two DSPs, hence each DSP process up to 2500 $\mathrm{Mb} / \mathrm{s}$ [2]. Hence the computing time is an important constraint and the RODs must implement a fast algorithm to reconstruct energy accurately in $10 \mu \mathrm{s}$.

Manuscript received June 5, 2005; revised April 24, 2006. This work was supported by the Spanish Ministerio de Educación y Ciencia.

E. Fullana, V. Castillo, C. Cuenca, A. Ferrer, E. Higon, J. Poveda, A. RuizMartinez, B. Salvachua, C. Solans, and J. Valls are with the Instituto de Fisica Corpuscular, IFIC (UV-CSIC), E-46071 Valencia, Spain (e-mail: Esteban.Fullana@ific.uv.es)

J. Castelo, C. Iglesias, and A. Munar were with the Instituto de Fisica Corpuscular, IFIC (UV-CSIC), E-46071 Valencia, Spain.

R. Teuscher was with the University of Chicago, Chicago IL 60637 USA. $\mathrm{He}$ is now with the Canadian Institute of Particle Physics (IPP), University of Toronto, Toronto, ON M5S 1A7, Canada (e-mail:teuscher@physics.utoronto. ca).

Color version of Figs. 1-4 are available online at http://ieeexplore.ieee.org. Digital Object Identifier 10.1109/TNS.2006.877267
We present an algorithm, so called Optimal Filtering (OF) algorithm, to reconstruct amplitude, time information and pedestal from a PM signal. The algorithm also provides an online quality check in order to decide in real time whether raw data should be added to the output stream in order to perform posterior analysis. The algorithm was first developed for liquid ionization calorimeters [3], its performance in hadronic calorimeters is the aim of the present analysis.

\section{THEORETICAL BACKGROUND}

Let's define $\boldsymbol{g}$ as a set of values of the pulse shape function of the signal, $g(t)$, noise free and normalized to one in amplitude. The $\boldsymbol{g}$ values are taken at times $t_{i}$, the time interval of which must be equal to the sampling period. The samples can thus be expressed as:

$$
S_{i}=p+A g\left(t_{i}+\tau\right)+n_{i}
$$

where $S$ represents the digital samples, $A$ is the true amplitude of the signal, $\tau$ accounts for a phase between the pulse shape factors and the samples, $n_{i}$ is the noise contribution and $p$ is the pedestal.

We can develop $g$ in a Taylor's series as:

$$
\begin{aligned}
S_{i} & \simeq p+A g\left(t_{i}\right)+A \tau g^{\prime}\left(t_{i}\right)+n_{i} \\
& =p+A g_{i}+A \tau g_{i}^{\prime}+n_{i} .
\end{aligned}
$$

Notice that the algorithm uses a first order aproximation for the phase between the samples $S$ and the pulse shape factors $g$, as the phase, $\tau$, tends to zero as more accurate the reconstruction. Therefore it is important to calculate the $\boldsymbol{g}$ components as close as possible from the positions of the samples within the signal.

Let us define now three quantities:

$$
u=\sum_{i=1}^{n} a_{i} S_{i}, \quad v=\sum_{i=1}^{n} b_{i} S_{i}, \quad w=\sum_{i=1}^{n} c_{i} S_{i}
$$

where $n$ is the number of samples and $\boldsymbol{a}, \boldsymbol{b}$ and $\boldsymbol{c}$ are free parameters of the algorithm called OF weights.

We set now two conditions:

- The expected values of $u, v$ and $(w\langle u\rangle,\langle v\rangle$ and $\langle w\rangle)$ for $m$ events of equal amplitude, time and pedestal must be equal to $A, A \tau$ and $p$ respectively.

- The distributions of $u, v$ and $w$ values are broaded by the noise. We require the parameters $\boldsymbol{a}, \boldsymbol{b}$ and $\boldsymbol{c}$ to be calculated so that they minimize the $u, v$ and $w$ variances. 
The theoretical development of the algorithm assumes a stationary noise, i.e., the statistical averages of the noise terms must be time independent, otherwise the algorithm is not valid.

With these conditions and using the Lagrange multipliers method - to minimize a function imposing constraints - we obtain three sets of $n+3$ equations and $n+3$ unknowns. The three systems of equations are linear (due to the Taylor's expansion) and their solutions are the parameters $\boldsymbol{a}, \boldsymbol{b}$ and $\boldsymbol{c}$ (in [3] we find the details of the resolution for $n+2$ equations and $n+2$ unknows):

$$
\left(\begin{array}{ccccccc}
R_{11} & R_{12} & \ldots & R_{1 n} & g_{1} & g_{1}^{\prime} & 1 \\
R_{21} & R_{22} & \ldots & R_{2 n} & g_{2} & g_{2}^{\prime} & 1 \\
\vdots & \vdots & \ddots & \vdots & \vdots & \vdots & \vdots \\
R_{n 1} & R_{n 2} & \ldots & R_{n n} & g_{n} & g_{n}^{\prime} & 1 \\
g_{1} & g_{2} & \ldots & g_{n} & 0 & 0 & 0 \\
g_{1}^{\prime} & g_{2}^{\prime} & \ldots & g_{n}^{\prime} & 0 & 0 & 0 \\
1 & 1 & \ldots & 1 & 0 & 0 & 0
\end{array}\right)\left(\begin{array}{c}
a_{1} \\
a_{2} \\
\vdots \\
a_{n} \\
\lambda \\
\kappa \\
\epsilon
\end{array}\right)=\left(\begin{array}{c}
0 \\
0 \\
\vdots \\
0 \\
1 \\
0 \\
0
\end{array}\right)
$$

where $g_{i}$ and $g_{i}^{\prime}$ are the values of the pulse shape and its derivative respectively, $a_{i}$ are the OF weights (the system for $\boldsymbol{b}$ and $\boldsymbol{c}$ weights is identical only the independent term changes), $\lambda, \kappa, \epsilon$ are the Lagrange multipliers and $R_{i j}$ are terms of the noise autocorrelation matrix which can be calculated by:

$$
R_{i j}=\frac{\sum\left(n_{i}-\left\langle n_{i}\right\rangle\right)\left(n_{j}-\left\langle n_{j}\right\rangle\right)}{\sqrt{\sum\left(n_{i}-\left\langle n_{i}\right\rangle\right)^{2} \sum\left(n_{j}-\left\langle n_{j}\right\rangle\right)^{2}}}
$$

where $n_{i}$ are noise samples.

Therefore, the OF algorithm reconstructs the amplitude, phase and pedestal information from weighted sums of the samples:

$$
A=\sum_{i=1}^{n} a_{i} S_{i}, \quad A \tau=\sum_{i=1}^{n} b_{i} S_{i}, \quad p=\sum_{i=1}^{n} c_{i} S_{i} .
$$

In order to check the quality of the reconstruction we define a quality factor, $\chi$ :

$$
\chi=\sum_{i=1}^{n} \operatorname{ABS}\left(\left(S_{i}-p\right)-A g_{i}\right) .
$$

We use the absolute value formula instead of the square because is faster to implement in the ROD DSPs. Notice that when the computing time is limited, as in the TileCal ROD, the $p$ calculation is not necessary, one sample without signal is enough as the $\chi$ factor pretends to be only an estimator of the quality of the reconstruction.

\section{IMPlEMENTATION IN THE AtLAS TILE CALORIMETER}

The weights are calculated offline and used online event by event. In order to calculate the weights, the noise and the shape function must be understood.

The noise autocorrelation matrix is calculated by forcing triggers with no signal. In this analysis the noise autocorrelation matrix is set to the unitary matrix due to the small correlation

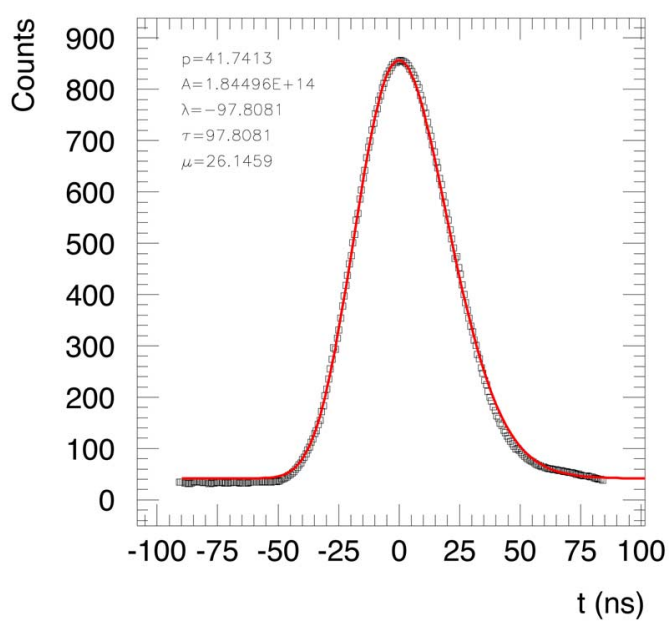

Fig. 1. Pulse shape reconstructed with charge injection events (white squares) and function fitted to the reconstructed pulse shape (solid line). The figure prints the values of the fit parameters.

found in the TileCal electronics. However, once the LHC is operative the correlation must be corrected because of the effect of the pile-up produced in each collision at the LHC.

The pulse shape is reconstructed using the charge injection system which is part of the TileCal front-end electronics. This system injects charge directly to the shaper emulating a PM. The injected charges range between zero and $800 \mathrm{pC}$ and the injection start time ranges to cover the $25 \mathrm{~ns}$ sampling period. Therefore, the system sweeps all the signal range allowing us to obtain a set of numerical values which define the pulse shape. We fit an analytical function to the numerical values obtained from the charge injection system in order to eliminate the front-end electronics noise in the pulse shape. We use the function:

$$
\mathrm{SF}(t)=p+A\left(\frac{t-\lambda}{\tau}\right)^{\mu} \exp \left(-\mu \frac{t-\lambda}{\tau}\right)
$$

which fits the pulse shape of the TileCal PM signal. Fig. 1 plots the numerical values versus the time (white squares) and the function fitted (solid line) and prints the values of the parameters obtained after the fit. The samples $g_{i}$ are calculated from this function once it is normalized to one in amplitude.

The OF algorithm needs the samples to come within a narrow time interval from the OF weights reference time (set by the time positions where $\boldsymbol{g}$ are calculated). However if the events do not arrive synchronously at the detector the phase between the samples and the $\boldsymbol{g}$ components changes event by event. The problem is solved by applying the proper weights for each event according to the position of the samples in the signal. In order to do that we calculate 25 sets of weights, one for each reference time between -25 and $25 \mathrm{~ns}$ in steps of $1 \mathrm{~ns}$ sweeping all the signal. The problem becomes thus to find out the position of the samples in the signal in order to choose the appropiate weights. The phase information provided by the OF algorithm can be used to start an iteration process. The convergence criteria is set by requiring the relative phase of the last iteration 

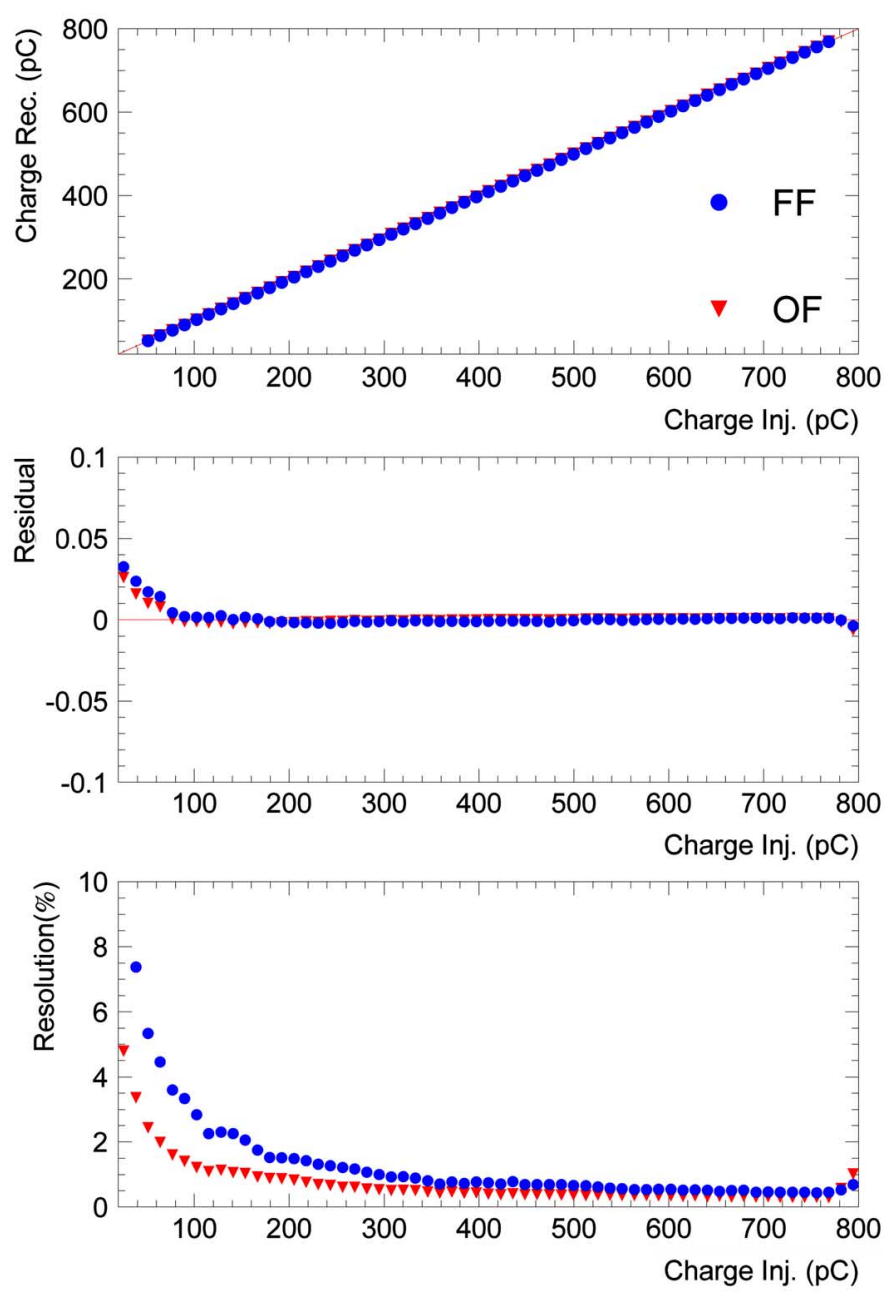

Fig. 2. Amplitude reconstruction for Optimal Filtering algorithm (OF) and Flat Filtering algorithm (FF) in charge injection events.

to be lower than half the time step between set of weights. The iteration process is limited by the computing time available, in our case the synchronism of ATLAS-LHC prevents us to implement iterations. However the arrival of the events in the set-up from which we acquired the data used in this analysis was asynchronous, hence we implement iterations.

\section{RESULTS}

We test the algorithm using two types of data, charge injection data and physics data. The injected charge in the charge injection system ranges between zero and $800 \mathrm{pC}$ and the injection start time ranges to cover the $25 \mathrm{~ns}$ sampling period. These characteristics allow to test the algorithm performance in both, amplitude and time, for all the charges availables.

Fig. 2 shows the results of the algorithm for amplitude reconstruction. The results are compared with the Flat Filtering algorithm (FF) which consists in a plain sum of the samples. The top plot shows the reconstructed charge versus the injected charge for the whole range of charges, both in picocoulombs. For each charge injected we reconstruct $\sim 200$ events sweeping all the phase range. The points represent the average of the distribution of the reconstructed charge for each injected charge.
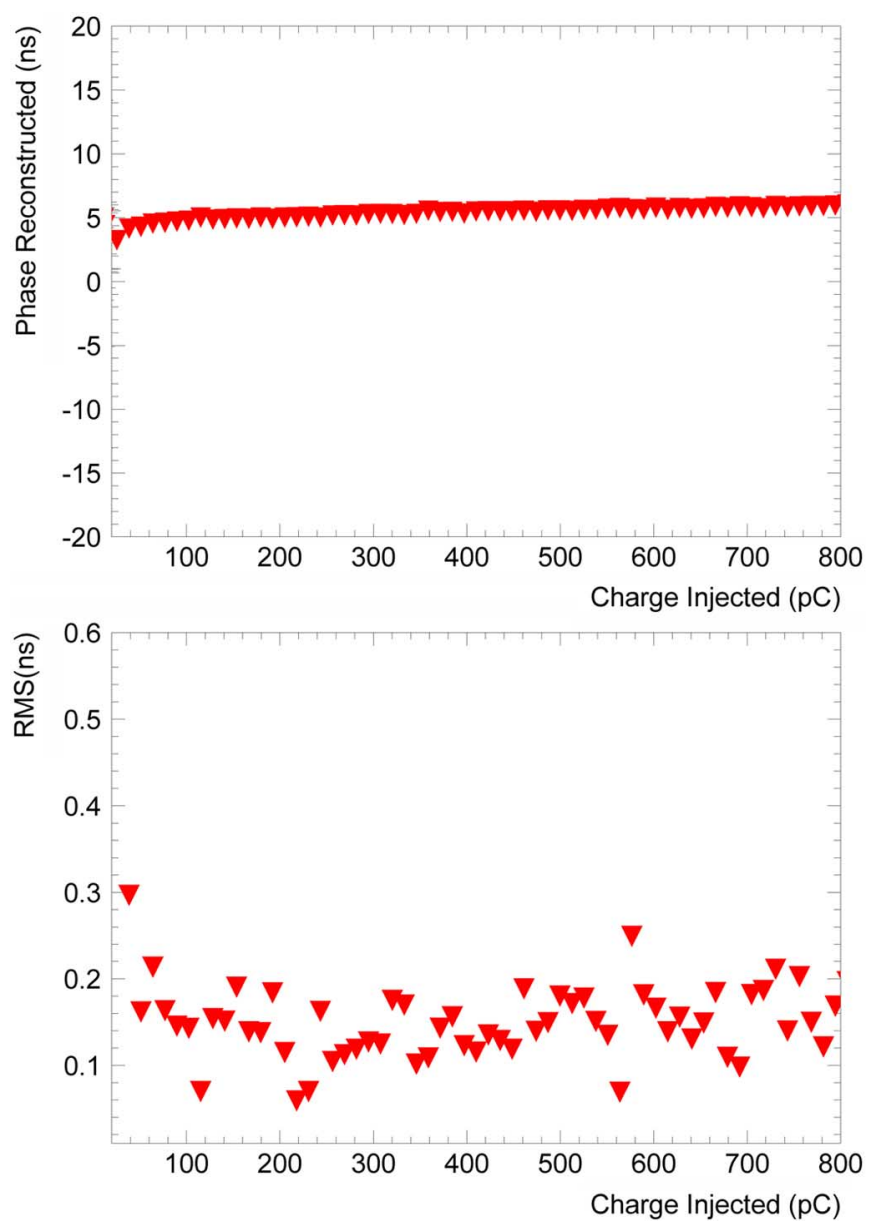

Fig. 3. Time reconstruction for Optimal Filtering algorithm in charge injection events.

The middle plot represents the residual of the points to the line which bisects the graph. Both plots show that both algorithms output on average a correct reconstructed charge. The bottom plot shows the resolution of the reconstruction versus the injected charge. The resolution is defined as the ratio between the standard deviation and the average of the distribution of the reconstructed charge. The plot shows the difference between the FF algorithm and the OF one. The resolution at high injected charges is similar for both algorithms, however as the injected charge decreases the OF algorithm plots better resolution. This is a consequence of the OF basic concepts, the algorithm is designed to minimize the noise impact on the resolution which is more important at low charges where the signal to noise ratio is small.

Fig. 3 shows the plots for the time reconstruction of the $\mathrm{OF}$ algorithm. The phase between the samples and the $\boldsymbol{g}$ values was fixed in $5 \mathrm{~ns}$. The top plot shows the average of the reconstructed phase distribution and the bottom plot shows its standard deviation both versus the injected charge for the whole range of injected charges. Notice that the phase is well reconstructed for the whole range of charges having an accuracy of $200 \mathrm{ps}$.

Fig. 4 shows the plots for the quality factor, $\chi$, of the OF algorithm. The plot shows that for most of the injected charge range the reconstruction is good only at very low injected charges the reconstruction starts to be less reliable. 

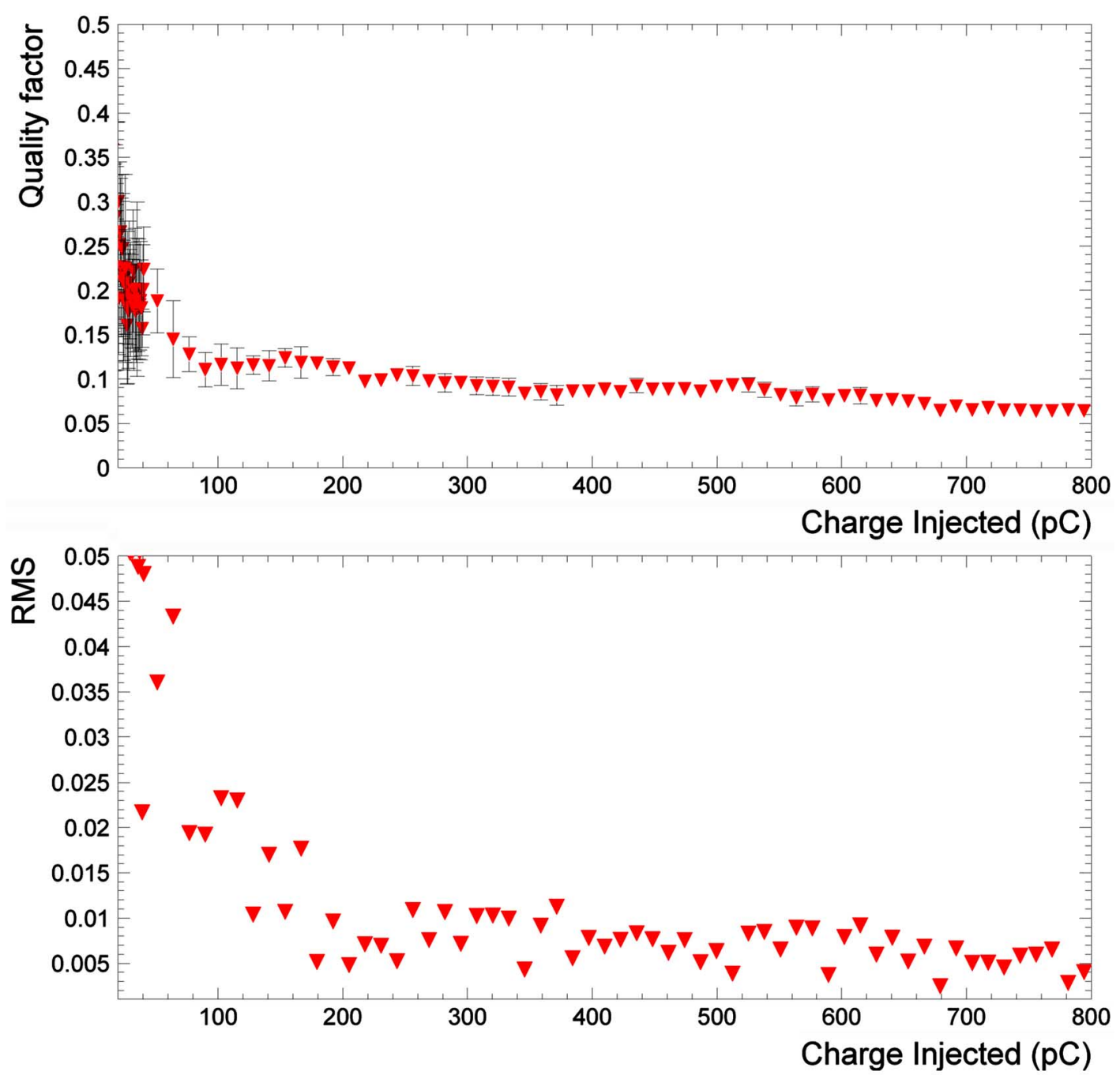

Fig. 4. Average of the quality factors of the Optimal Filtering algorithm versus the injected charge.

Notice that the time reconstruction helps to reject backgrounds and the quality factor is important to monitor online the quality of the reconstruction and to take online decisions about the data to be sent to the next step in the acquisition chain.

For the physics data we use pions and electrons of several energies. The data was taken during testbeam periods using the SPS accelerator at H8 CERN facility. The total energy deposited in the calorimeter is computed by summing the amplitude reconstructed in all the channels multiplied by a calibration constant. We fit a Gaussian distribution to the energy deposited in the calorimeter. The resolution of the calorimeter is defined here as the ratio between the sigma and the average of the distribution.

Fig. 5 shows the resolutions obtained with the OF and FF algorithm versus the momentum of the incident electron. As in the charge injection case the improvement of the $\mathrm{OF}$ algorithm is manifested at low energies where the signal to noise ratio is small and the noise degrades significantly the resolution. Fig. 6 shares the same result, now the resolutions are in general worse than in the electron case due to the intrinsic fluctuations of the shower developed by the pions but again the OF algorithm improves the resolution at low energies. Notice than the differences between $\mathrm{FF}$ and $\mathrm{OF}$ measurements in the TileCal resolution are

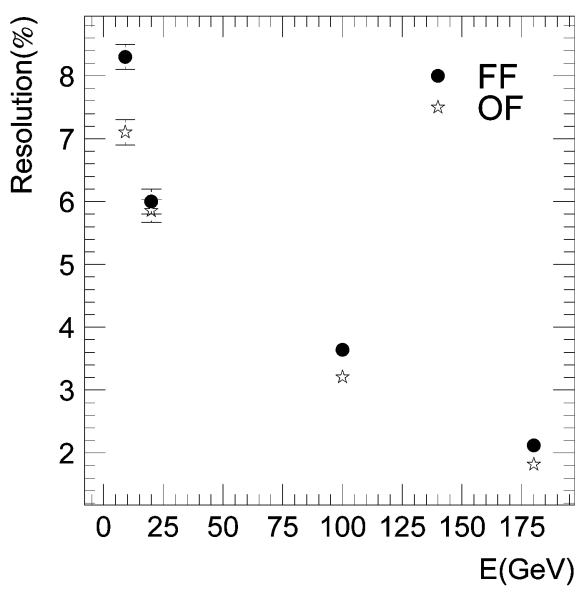

Fig. 5. Energy resolution of the TileCal detector obtained with the Optimal Filtering (OF) and Flat Filtering (FF) algorithm versus the momentum of the incident electrons.

not very significant in general for physics events. The important point is that $\mathrm{OF}$ does show significant improvement in the regions where the noise is degrading the resolution which is a 


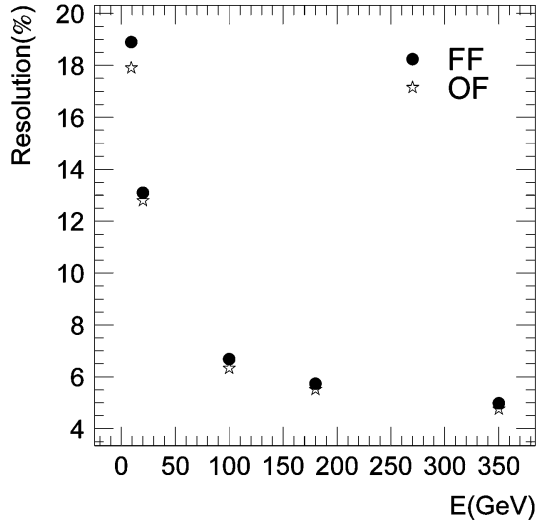

Fig. 6. Energy resolution of the TileCal detector obtained with the Optimal Filtering (OF) and Flat Filtering (FF) algorithm versus the momentum of the incident pions.

promising result facing the future overall noise increase due to LHC pile up events.

\section{CONCLUSION}

The Optimal Filtering algorithm has been tested satisfactorily in two types of data. For the data obtained with charge injection runs the reconstruction is correct for both amplitude and time. At the same time the algorithm improves the resolution, compared with plain filtering algorithms, when the signal to noise ratio is small. This result is shared in physics runs taken during physics calibration periods of the detector. Therefore the results are promising for the $\mathrm{OF}$ algorithm to be a good candidate to reconstruct online the energy of the Tile Calorimeter when the LHC will be operative.

\section{ACKNOWLEDGMENT}

The authors would like to acknowledge the contribution of B. Cleland for his wise advice and for sharing his wide knowledge about signal analysis, and express gratitude to R. Leitner, B. Stanek, T. Davidek and the people involved in the TileCal detector, especially the ones who contributed to the data acquisition and testbeam calibration period. The authors also thank C. Bohm for the encouragement to present this work.

\section{REFERENCES}

[1] C. Atlas, Tile Calorimeter Tech. Design Rep., CERN, Geneva, Switzerland, CERN/LHCC 96-42, 1996

[2] J. Castelo, "TileCal ROD hardware and software requirements," ATLAS Internal Note, 2005, CERN-ATL-TILECAL-2005-003.

[3] W. E. Cleland and E. G. Stern, "Signal processing considerations for liquid ionization calorimeters in a high rate environment," Nucl. Instrum. Methods Phys. Res. A, vol. A338, p. 467, 1994. 